\title{
Causes de la monodominance de l'essence Cynometra alexandri C.H. Wright (Eggeling 1947; Hamilton 1981), dans la forêt de Banana (Mambasa/Ituri/R.D. Congo)
}

\begin{abstract}
Basile LUSE Belanganayi ${ }^{1}$
basileluse@yahoo.fr

${ }^{1}$ Université Officielle de Ruwenzori, Faculté des Sciences, Butembo, République Démocratique du Congo.

\section{RESUME}

La structure diamétrique et la répartition spatiale d'une essence tolérante à l'ombre à graines pesantes, de la forêt tropicale humide, Cynometra alexandri, ont été étudiées respectivement au travers le prélèvement de Diamètre à Hauteur de Poitrine (DHP) et de la fonction K de Ripley transformée. L'étude a été menée en République Démocratique du Congo, dans la localité de Banana, territoire de Mambasa, Province de l'Ituri, sur une parcelle de $120 \times 500$ $\mathrm{m}$, où 101 individus adultes ont été recensés, mesurés et cartographiés. La répartition spatiale s'est révélée agrégée dans cette parcelle où $C$. alexandri est monodominant. En revanche, la structure diamétrique se présente en cloche déformée. Pour finir, des traits de vie de $C$. alexandri pouvant expliquer sa monodominance ont été analysés et un certain lien entre la répartition agrégée, la monodominance et le climax, a été conjecturé.
\end{abstract}

Mots clés : Cynometra alexandri, Répartition spatiale, structure diamétrique, Translation d'axes, Fonction de Ripley, monodominance.

\begin{abstract}
The diametric structure and the spatial distribution of a shade tolerant specie with heavy seeds, of tropical-rain forest, Cynometra alexandri, have been studied respectively through Diameter at Breast Height (DBH) taking and transformed K Ripley function. The study was performed in Democratic Republic of Congo, in Banana village, Mambasa territory, Ituri province on a $120 \times 500 \mathrm{~m}$ plot, in which 101 adult individuals was identified, measured and mapped. The spatial distribution was revealed aggregated in this parcel where C. alexandri is monodominant. On the other hand, the diametric structure was like a deformed bell. In finish, life traits of $C$. alexandri capable to explain its monodominance have been analysed then, a certain link between aggregated distribution, monodominance and climax, has been conjectured.
\end{abstract}

Key words: Cynometra alexandri, spatial distribution, diametric structure, axis change, Ripley Function, monodominance. 


\section{INTRODUCTION}

Certaines espèces ligneuses des forêts tropicales humides, sont tolérantes à l'ombre du sous-bois, y germent et entrent en phase d'attente de meilleurs conditions lumineuses pour continuer leur croissance, tandis que d'autres ne peuvent germer qu'en présence d'une lumières suffisante (Whitmore, 1978 ; Alexandre, 1989). Plusieurs arbres appartenant à des espèces tolérantes à l'ombre et possédant des graines pesantes présentent des mécanismes de dispersion qui n'éloignent guère les graines des adultes producteurs. Si ces graines ne possèdent pas de caractéristiques permettant d'attirer des espèces animales ou microbiennes à s'en nourrir, les plantules et jeunes plants pourront très facilement avoir la chance de pousser autour des leurs parents (Torti et al., 2001). Ce mécanisme se répétant dans le temps aboutit généralement à une agrégation des individus de cette espèce (Boyemba, 2011); l'agrégation issue de ce mécanisme peut aboutir à une monodominance d'une vaste étendue de forêt si l'espèce concernée possède certains traits de vie particuliers.

Des exemples des larges espaces dominés par des grands arbres appartenant à une seule espèce ou à quelques espèces seulement sont rapportés par plusieurs études effectué en forêt tropicale (Torti et al 2001; Wolley et al.; Van der Velden et al. 2014; Peh et al., 2014). Cependant, les forêts tropicales humides demeurent parmi les communautés végétales sauvages les plus diversifiées (Blanc et al. 2003 ; Boyemba 2011). Cette grande richesse spécifique s'avère être en contraste avec la monodominance observée chez certaines espèces. En outre, certaines espèces présentent la monodominance dans certains sites d'une forêt alors qu'elles ne la présentent pas dans d'autres sites de la même forêt (Lomba, 2011). Au-delà de ce constat, les questionnements sont multiples, en dépit de nombreuses tentatives d'explications, d'où la nécessité de multiplier les études pouvant peu à peu élucider ces questions. C'est dans cet ordre d'idée que nos objectifs ont été de déterminer la structure diamétrique de l'espèce Cynometra alexandri dans la forêt hétérogène de Banana, de définir le type d'agrégation de ses individus et enfin, de rechercher des éventuels liens entre ses traits de vie, sa structure diamétrique, sa répartition spatiale et sa monodominance.

\section{MATERIELS ET METHODES}

\subsection{Station d'étude}

La parcelle d'étude est située dans la forêt tropicale humide de la localité de Banana, à environ $25 \mathrm{~km}$ de la cité de Mambasa, dans le territoire du même nom, district de l'Ituri, 
Province Orientale, République Démocratique du Congo. Elle s'étend sur 6 ha, entre les parallèles $1,402330^{\circ} \mathrm{N}-1,406604^{\circ} \mathrm{N}$ et les méridiens $28,59140^{\circ} \mathrm{E}-28,896990^{\circ} \mathrm{E}$ (fig.1). Le climat y est du type tropical humide à très courte saison sèche. L'altitude est relativement basse et varie entre 844,6 et 863,5 mètres.

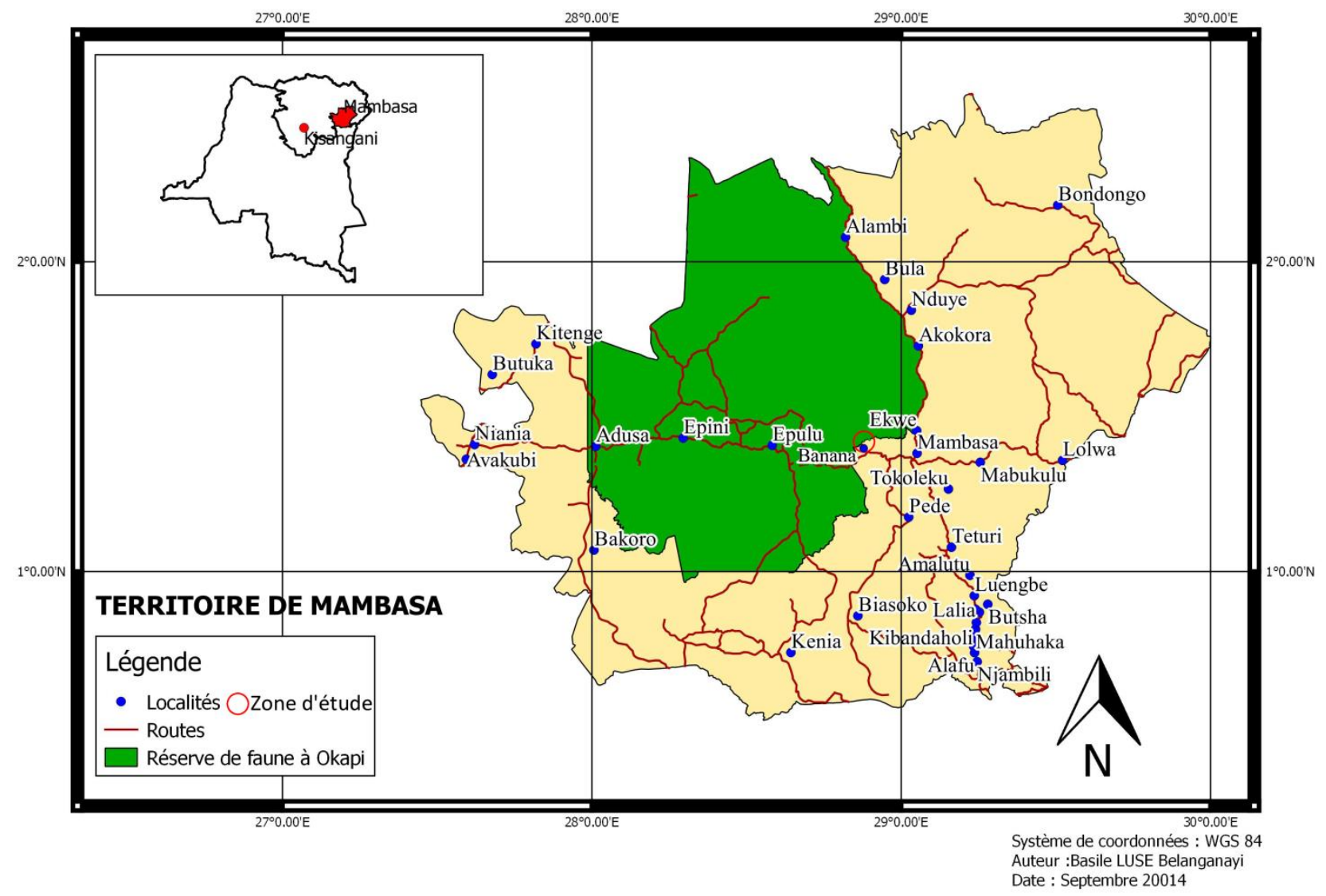

Fig. 1 : Région d'étude.

\subsection{Caractéristiques de l'espèce étudiée}

L'espèce Cynometra alexandri (fig. 2) appartient à l'Ordre des Fabales, Familles des Fabaceae, Sous-famille des Caesalpinioideae, Genre Cynometra.

C'est un arbre forestier bas-guinéo-congolais, toujours vert, spontané (Lejoly et al., 2010), au bois très dur et très inflammable, au tronc muni de grands empattements basilaires, à cime étalée.

Il est répandu dans tout le District Forestier Central jusqu'en Ubangi-Uele, ainsi que dans la partie de la dorsale du Kivu. Il se rencontre aussi dans le District Forestier du Lomami (Bas-Katanga) et dans celui de la plaine de la Ruzizi. C'est une essence de la forêt ombrophile équatoriale de terre ferme, poussant aux altitudes ne dépassant guère $1400 \mathrm{~m}$ (Ndele, 1988). En dehors, de la République Démocratique du Congo, elle est connue dans l'ouest de l'Ouganda (Hart et al., 1989 ; Sheil \& Salim, 2004 ; Zziwa et al., 2009) et dans l'ouest de la Tanzanie (Cobbinah \& Obeng, 2011). Ses forêts monodominantes sont considérées comme étant des végétations climaciques (Osmaston, 1959). 
C. alexandri est une plante mellifère dont l'écorce sert à faire des paniers et la sève à fabriquer un poison à flèche. La poudre d'écorce est utilisée dans le traitement de la lèpre et des autres maladies cutanées. Les chenilles qui se nourrissent de ses feuilles sont récoltées pour la consommation ou pour servir d'appât de pêche (Cobbinah \& Obeng, 2011).
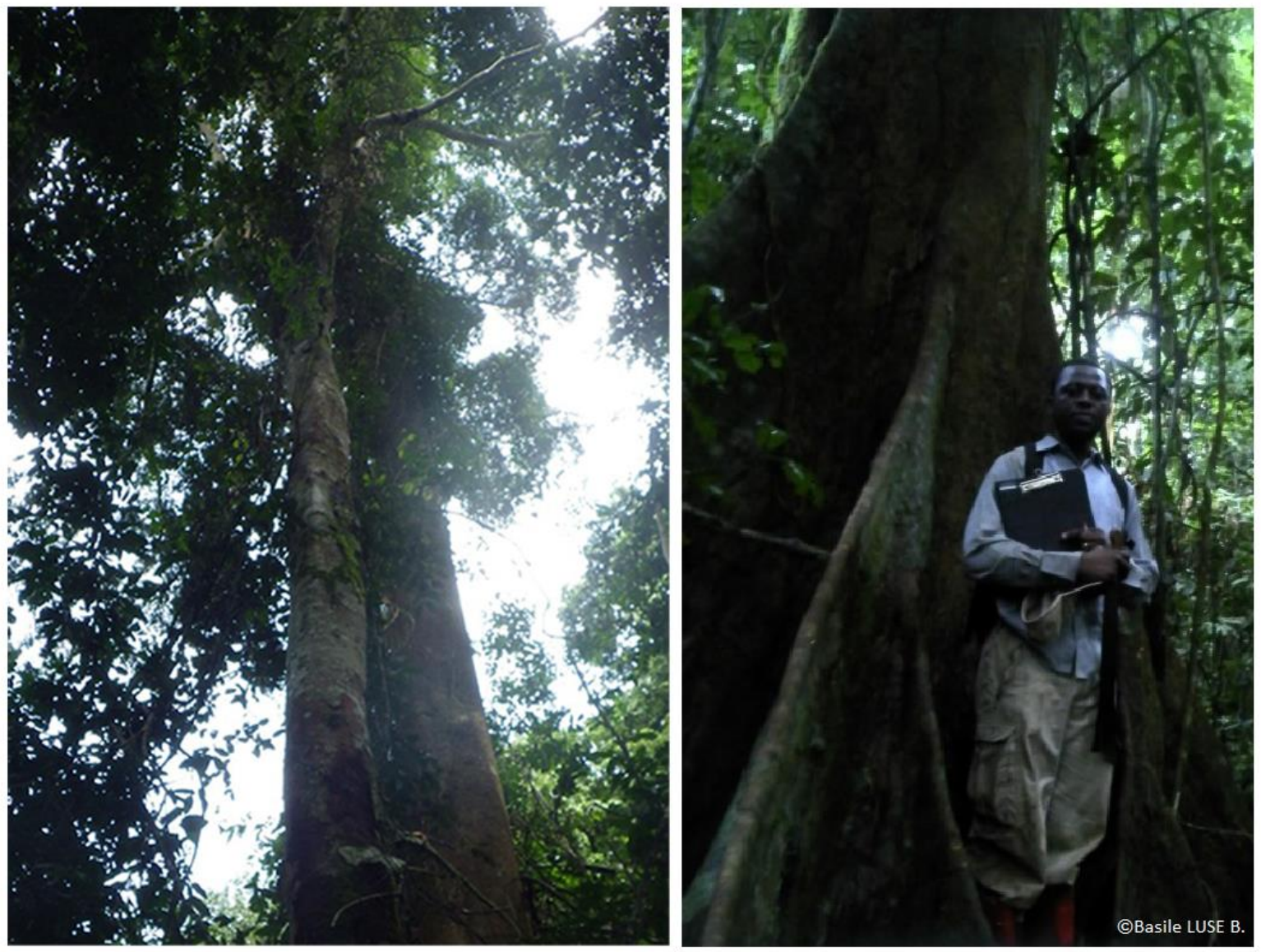

Fig. 2 : Houppier et fût de Cynometra alexandri (dans la parcelle d'étude).

\subsection{Délimitation de la parcelle d'étude et layonnage}

La superficie du dispositif d'inventaire, orienté Nord-Sud (MECNEF, 2007), était de 6 ha soit $120 \mathrm{~m} \times 500 \mathrm{~m}$. Nous l'avons subdivisé en 4 placettes de 1,5 ha de superficie chacune, soit $30 \mathrm{~m} \times 500 \mathrm{~m}$. La figure 3 donne un aperçu de ladite parcelle. 


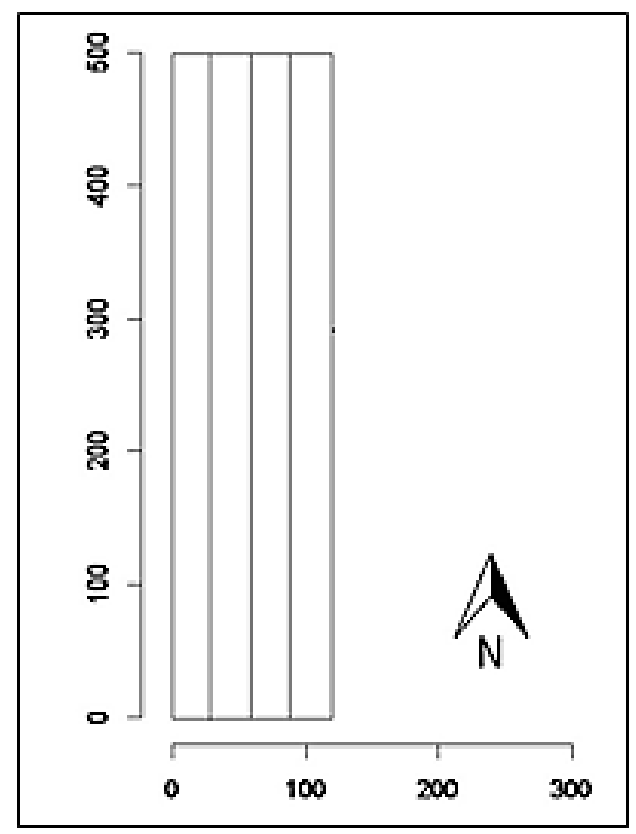

Fig. 3 : Parcelle d'inventaire.

\subsection{Comptage d'arbres}

L’inventaire des individus de Cynometra alexandri a été réalisé en se basant sur la prise des positionnements en abscisses (x) et en ordonnées (y) des individus de l'espèce. Nous avions à l'aide d'un GPS garmin 64s worldwide, marqué les coordonnées géographiques de tous les individus de DHP $\geq 10 \mathrm{~cm}$, appartenant à l'espèce, placette par placette. Les individus de DHP $\geq 10 \mathrm{~cm}$ des autres espèces ligneuses ont également été répertorié enfin de déterminer la densité de la parcelle d'étude.

Les coordonnées géographiques (longitude et latitude) des arbres de l'espèce $C$. alexandri inventoriés, assimilant ces derniers à des points dans un plan, ont été converties en coordonnées projetées, exprimées en mètre, grâce au logiciel QGIS. Puis, une translation d'axes a été effectué pour ramener ces points à un système tel que l'origine des axes $(0,0)$ soit située au point de coordonnées géographiques 28,895914 ${ }^{\circ}$ Est (Soit 710943,5 m Est) et $1,402330^{\circ}$ Nord (Soit 155085,4 m Nord), correspondant à la vraie origine des axes sur terrain.

Les formules (1.1) ont été d'usage pour cette translation d'axes donnant lieu aux nouvelles coordonnées des arbres, utilisées dans l'analyse de la répartition spatiale :

$$
\left\{\begin{array}{l}
x^{\prime}=x-a \\
y^{\prime}=y-b
\end{array}\right.
$$

Avec :

$\left(\mathrm{x}^{\prime}, \mathrm{y}^{\prime}\right)$, les nouvelles coordonnées d'un point $\mathrm{P}$ 
(x, y), les anciennes coordonnées du point $\mathrm{P}$.

(a, b), les anciennes coordonnées de la nouvelle origine des axes, dans le cas échéant $\quad(a, b)=(710943,5 ; 155085,4)$.

L'ensemble de ces nouvelles coordonnées des arbres a constitué un "semis de points" sur lequel nous avons effectué la caractérisation de la structure spatiale des individus de Cynometra alexandri dans la parcelle d'étude en faisant le calcul de la fonction de Ripley transformée (par usage du logiciel R) comme l'ont fait Pelissier (1995), Picard (1999) et Traissac (2003) dans leurs travaux.

\section{RESULTATS ET DISCUSSION}

\subsection{Abondance des individus}

Les figures 4 et 5 donnent respectivement la répartition spatiale en coordonnées géographiques et la répartition spatiale en coordonnées cartésiens (après translation d'axes) des individus de Cynometra alexandri inventoriés. Pour la carte en coordonnées géographiques la parcelle est délimitée par les droites de coordonnées suivantes : $\mathrm{X}=$ $28,59140^{\circ} ; \mathrm{Y}=1,402330^{\circ} ; \mathrm{X}=28,896990^{\circ} ; \mathrm{Y}=1,406604^{\circ}$.

Nous avons inventorié 101 individus de C. alexandri sur un total de 164 individus toutes les espèces ligneuses confondues, dans une parcelle de 6 ha. Ce qui donne une densité en $C$. alexandri de 16,83 individus /ha (contre une densité totale de 27,33 individus/ha). Cette valeur semble relativement faible pour une population ligneuse située en zone tropicale humide où la densité de tige varie entre 300 et 700 tiges/ha, si l'on considère les tiges à partir de $10 \mathrm{~cm}$ de diamètre (Dupuy et al., 1998). Cependant, ceci est tout à fait justifiable par le fait que les forêts tropicales humides qui se composent d'arbres de fortes dimensions, comme il en était le cas avec celle où l'on a travaillé (Voir le paragraphe structure diamétrique), ont un nombre de tiges qui va décroissant au fur et à mesure que le diamètre augmente (Dupuy et al., 1998). Rollet, 1974 définit une marge de 20-30 tiges/ha pour une forêt dominée par des arbres de DBH supérieur à $55 \mathrm{~cm}$, ce qui est en conformité avec nos observations. 


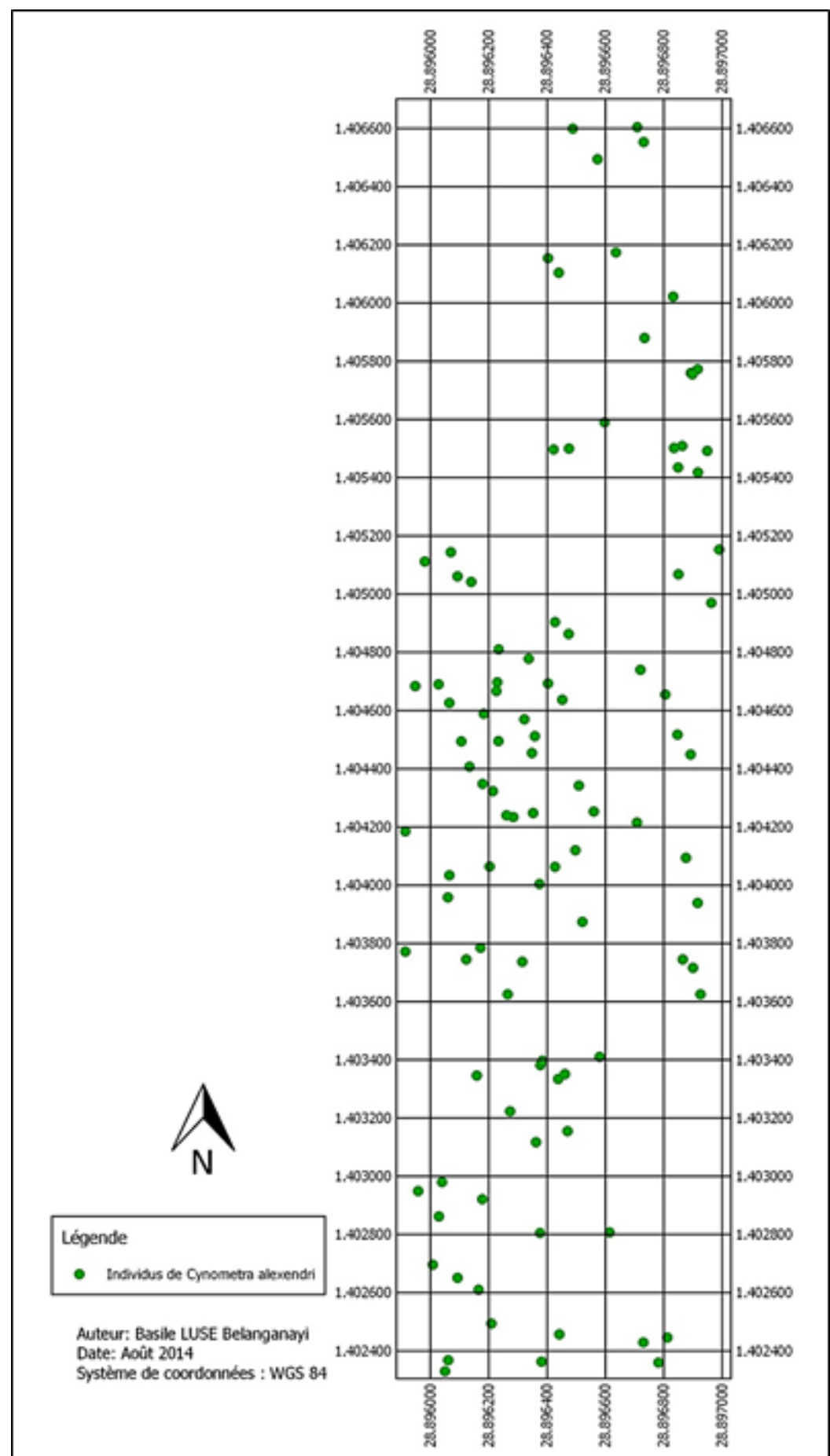

Fig. 4: Répartition spatiale réelle des individus de Cynometra alexandri dans la parcelle d'étude. 


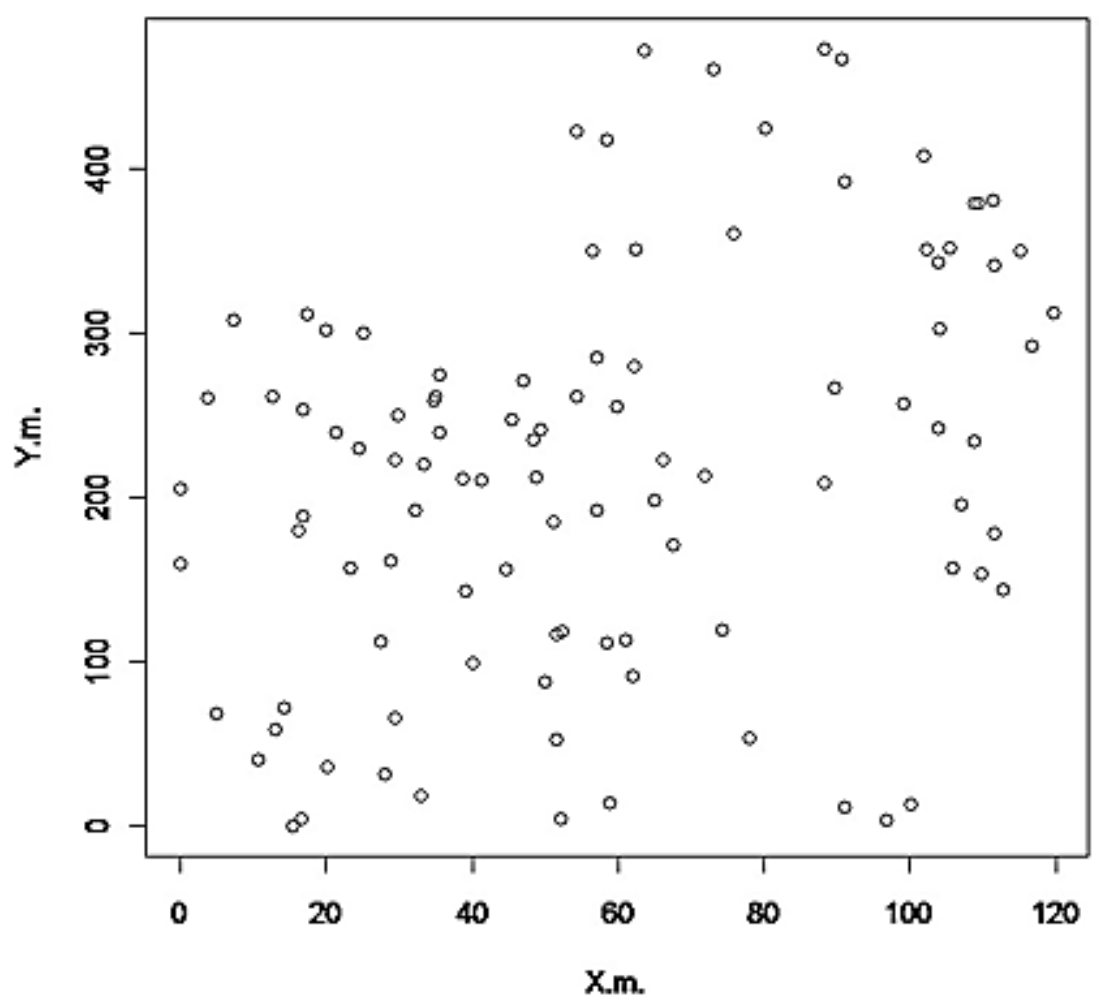

Fig. 5: Répartition spatiale des individus de Cynometra alexandri en coordonnées cartésiens.

\subsection{Structure diamétrique}

Dans la parcelle d'étude, l'essence Cynometra alexandri présente une structure diamétrique (Fig. 6) qui, a vu de nez, est irrégulière mais qui après analyse, se présente comme une structure en cloche déformée par une réduction d'effectif dans les classes de 50 $60 \mathrm{~cm}$ et $60-70 \mathrm{~cm}$. La classe diamétrique de $10-20 \mathrm{~cm}$ est complètement dépourvue et il y a absence totale d'individus de $\mathrm{DBH}<10 \mathrm{~cm}$, ce qui signifie que l'espèce ne se régénère pas. En effet, il est considéré que les espèces tolérantes à l'ombre présentent le plus souvent une structure diamétrique exponentielle décroissante (Dupuy et al., 1998). Les effectifs vont décroissant avec l'augmentation de diamètre à cause du fait que les graines peuvent germer sous l'ombre du sous-bois et que les plantules et les plants peuvent y rester aussi longtemps que possible attendant des conditions de lumière favorables (Puig et al., 1998). Cependant, une structure en cloche serait tout à fait normale, dans le cas de notre étude, si nous nous tenons au fait que les forêts à $C$. alexandri sont considérées comme climaciques (Osmaston, 1959) et que les forêts climaciques ne se régénèrent guère (Miller \& Spoolman, 2009). 


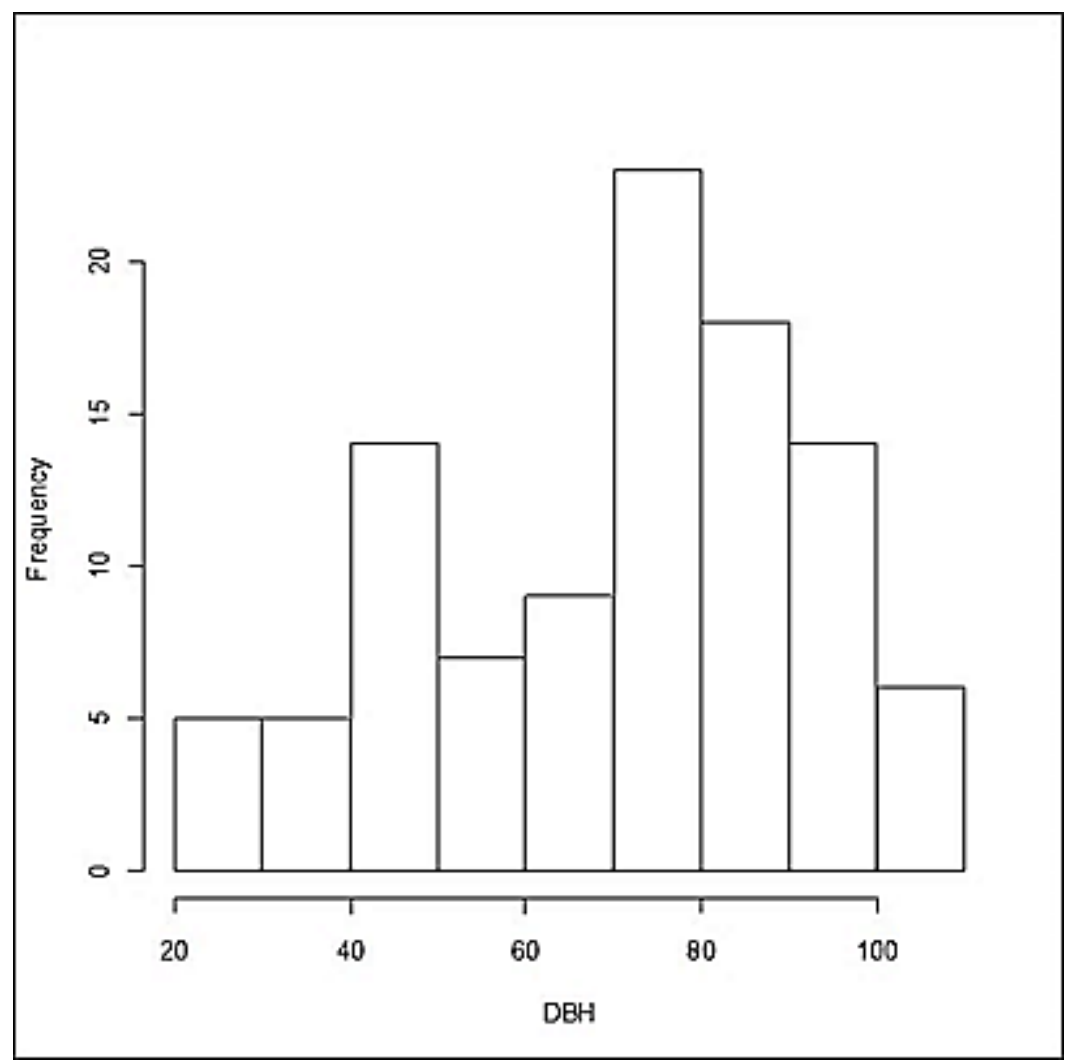

Fig. 6: Structure diamétrique des individus de Cynometra alexandri dans la parcelle d'étude.

\subsection{Répartition spatiale}

L'essence Cynometra alexandri présente une répartition spatiale principalement agrégée à courbe avec émarginations (Fig.7). On peut voir sur cette figure que la tendance de sa courbe d'agrégation est partout agrégée sauf aux distances $(r)$ comprises entre 0,5 et 9,5 m ainsi qu'entre 19 et $22 \mathrm{~m}$ où elle est aléatoire (p-value >0,05).

La distribution aléatoire aux petites distances respecte la théorie de Conell-Janzen qui stipule que le taux de mortalité est plus fort sous la couronne d'un arbre, où la densité des plantules est plus élevée (Hyatt et al., 2003). L'agrégation à des grandes distances confirme l'hypothèse de «colonisation» selon laquelle la dispersion accroit la probabilité de germination des graines dans de milieux-ouverts tels que les chablis (Puig et al., 1998). Par ailleurs, il est tout à fait normal que $C$. alexandri, une essence tolérante à l'ombre, effectuant une dispersion par barochorie présente une répartition spatiale agrégée. Car en effet, pour ce type d'essence, il n'y a pas mortalité des juvéniles due à l'absence de la lumière sous l'ombrage des parents (Torti et al., 2001). 
Les émarginations de la courbe (Fig. 7) se justifient par l'éloignement de quelques pieds à certaines distances. Eloignement qui pourrait avoir été causé par des animaux qui emportent les semences de la plante ou se nourrissent des juvéniles ou encore au déplacement de gousses par ruissèlement des eaux de pluie ou encore à l'activité des microorganismes qui décomposent les semences à certains endroits.

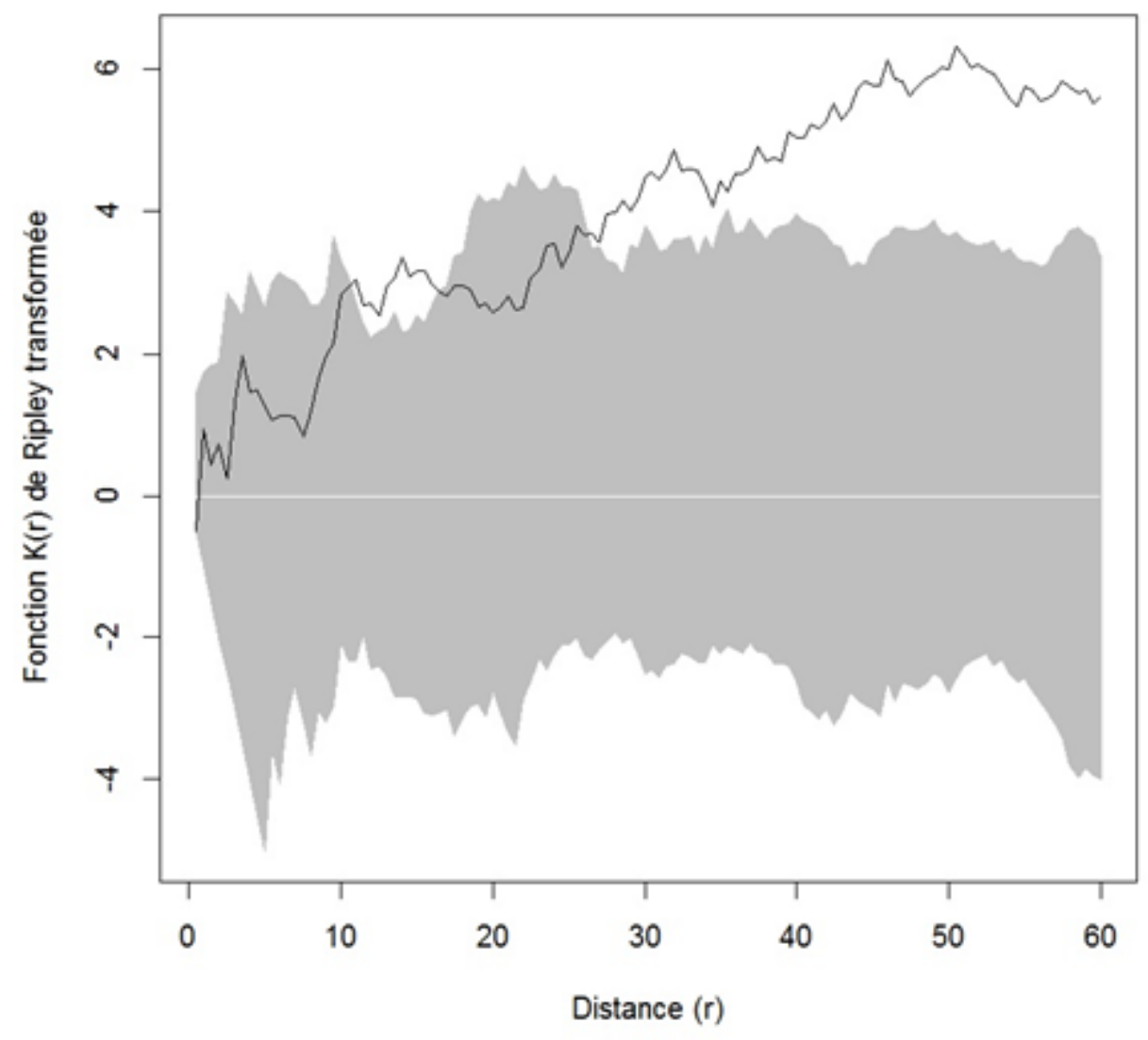

Fig. 7 : Fonction K(r) de Ripley transformée de l'étude.

\subsection{Monodominance}

Selon la définition donnée par Brookshire \& Thomas (2013), Cynometra alexandri est monodominant dans notre parcelle d'étude car représenté à plus de 60\% (précisément à 61,59 \%) dans la canopée.

On a défini un mode de vie et certaines propriétés aux espèces monodominantes dont principalement la barochorie, la tolérance à l'ombre, la production d'une litière abondante se décomposant lentement, la fermeture de la canopée et les associations ectomycorhizes, (Hart et al. 1989; Torti et al., 2001 ; Peh et al. 2014). Selon les auteurs précités, ces conditions rendent le milieu stressant pour d'autres espèces, pendant que les juvéniles de l'espèce monodominante, possèdent quant à eux des caractéristiques adaptées à ces conditions. 
Ces différents paramètres sont discutés ci-dessous en vue de tenter d'expliquer la monodominance de $C$. alexandri dans la forêt de Banana.

\subsubsection{Barochorie et tolérance à l'ombre}

Hart et al., (1989) affirment que les espèces dominante des forêts monodominantes sont toujours tolérantes à l'ombre et possèdent toujours des semences lourdes. Les mêmes chercheurs affirment que La monodominance indique que la forêt n'a pas subi de perturbations à grande échelle durant une longue période. Et qu'ultérieures à de grandes perturbations, les forêts monodominantes se régénèrent et se répandent, probablement, moins vite que les forêts mixtes.

En effet, l'essence Cynometra alexandri porte des graines barochores et est tolérante à l'ombre. Par ailleurs, nous avons constaté que notre parcelle d'étude a longtemps demeurée sans perturbations majeures (absence de champs et de signes véritables de déboisement) et que $C$. alexandri ne s'y régénère quasiment plus (absence totale des individus de diamètre inférieure ou égale à $20 \mathrm{~cm}$ ). Toutes ces considérations suffisent pour expliquer la contribution majeure de la barochorie et la tolérance à l'ombre de $C$. alexandri à l'établissement de sa monodominance.

\subsubsection{Production d'une litière abondante et fermeture de la canopée}

Torti et al. (2001) Soutiennent que la monodominance de Gilbertiodendron dewevrei est due, en partie, à la production d'une litière abondante et lente à la décomposition, empêchant aux semences de petites dimensions de pouvoir germer. Ils avancent ce même argument pour les espèces Mora excelsa, Mora gonggrijpii, Eperua falcate, Dryanobalanops aromatica et Shorea curtisii. Ils ajoutent que les adultes de l'espèce $G$. dewevrei modifient le régime lumineux en fermant la canopée. Cependant, Cynometra alexandri ne produit pas une litière abondante et son houppier ne ferme pas efficacement la canopée. D'où notre conviction que l'hypothèse d'abondance de la litière et celle de la fermeture de la canopée ne justifient pas suffisamment sa monodominance.

\subsubsection{Ectomycorhize}

Comme plusieurs espèces monodominantes établissent des ectomycorhizes, une hypothèse a été émise, soutenant que l'ectomycorhize est un facteur qui accroit la capacité compétitive de ses bénéficiaires ligneuses (McGuire et al., 2008). Selon Torti et al., 2001 Cynometra alexandri n'établit pas d'ectomycorhize. Par conséquent, celle-ci n'explique pas sa monodominance. 


\section{CONCLUSION}

La distribution spatiale de Cynometra alexandri, dans la parcelle d'étude est agrégée et en accord avec la théorie de Connell-Janzen et celle de colonisation. Sa structure diamétrique se présente comme une cloche déformée résultant d'une réduction de l'effectif des individus à diamètre compris entre 50 et $70 \mathrm{~cm}$. Bien que la structure en cloche ne soit pas communément observée chez les espèces tolérantes à l'ombre, elle est cependant une de leurs caractéristiques pendant le stade climacique qui correspond à une régénération non effective. Ainsi, vu que de tous les traits de vie avancés pour expliquer la monodominance, seules la barochorie et la tolérance à l'ombre se vérifient pour $C$. alexandri, nous pensons que le stade climacique de la forêt à $C$. alexandri de Banana est une réalité incontournable à cet effet. En fait, à ce stade, la compétition avec les espèces héliophiles étant absente, C. alexandri profite de son mode de dispersion agrégative pour établir sa monodominance. Mais nous ne savons pas grand chose de la compétition avec d'autres espèces tolérantes à l'ombre. C'est pourquoi nous pensons que des travaux portant sur l'implication du système d'enracinement de $C$. alexandri, qui pourrait dans une certaine mesure empêcher la régénération d'autres espèces ainsi que la nature biogéochimique du substrat doivent être menées pour compléter cette étude.

\section{Références}

Alexandre, D. (1989). Dynamique de la régénération naturelle en forêt dense de Côte d'Ivoire. Thèse d'Etat: Université Paris-Sud, Orsay, 102 p.

Blanc, L., Florès, O., Molino, J., Gourlet-Fleury, S., \& Sabatier, D. (2003). Diversité spécifique et regroupement d'espèces arborescentes en forêt guyanaise. Rev . For. Fr. $L V$, numéro spécial $2003: 131-146$.

Boyemba, F. (2011). Écologie de Pericopsis elata (Harms) Van Meeuwen (Fabaceae), arbre de forêt tropicale africaine à répartition agrégée. Thèse d'Etat: Université de Kisangani. $181 \mathrm{p}$.

Brookshire, E., \& Thomas, S. (2013). Ecosystem Consequences of Tree Monodominance for Nitrogen Cycling in Lowland Tropical Forest. PLoS ONE , 8 (7) : e70491 doi:10.1371/journal.pone.0070491.

Cobbinah, J., \& Obeng, E. (2011). Cynometra alexandri C.H.Wright. Lemmens, R.H.M.J., Louppe, D. \& Oteng-Amoako, A.A. (Editors). PROTA (Plant Resources of Tropical 
Africa), Wageningen, Netherlands. Consulté le juin 18, 2016, sur prota4u.org: http://www.prota4u.org/search.asp

Dupuy, B., De Madron, L., \& Petrucci, Y. (1998). Sylviculture des peuplements naturels en forêt dense humide Africaine : Acquis et recommandations. BOIS ET FORETS DES TROPIQUES , 257 (3) : 5-22.

Hart, T., Hart, J., \& Murphy, P. (1989). Monodominant and species-rich forests of the humid tropics: causes for their co-occurrence. The American Naturalist, 133 (5) : 613-633.

Hyatt, L. A., Rosenberg, M. S., Howard, T. G., Bole, G., Fang, W., Anastasia, J., et al. (2003). The distance dependence prediction of the Janzen-Connell hypothesis: a metaanalysis. Oikos , 103: 590-602.

Lejoly, J., Ndjele, M., \& Geerinck, D. (2010). Catalogue-Flore des plantes vasculaires des districts de Kisangani et de la Tshopo, (RD Congo), 4ème édition. Taxonomania, 30 (1-308, mai 2010), p.132.

Lomba, C. B. (2011). Systèmes d'agrégation et structures diamétriques en fonction des tempéraments de quelques essences dans les dispositifs permanents de Yoko et Biaro (Ubundu, P.O. R.D. Congo). Thèse d'Etat: Université de Kisangani. 335 p.

McGuire, K., Henkel, T., Granzow de la Cerda, I., Villa, G., Edmund, F., \& Andrew, C. (2008). Dual mycorrhizal colonization of forest-dominated tropical trees and the mycorrhizal status of non-dominant tree and liana species. Mycorrhiza, 18, 217-222.

Miller, G., \& Spoolman, S. (2009). Essential of Ecology, Fifth edition. . Belmont, CA 940023098, USA.: Brooks/Cole. P. 118.

Ministère de l'Environnement, Conservation de la Nature, Eaux et Forêts, R.D.Congo (MECNEF). (2007). Normes de l'inventaire d'exploitation. Kinshasa: MECNEF. 29 p.

Ndele, M.-B. (1988). Les éléments phytogéographiques endémiques dans la flore vasculaire $d u$ Zaïre. Thèse d'Etat: Université Libre de Bruxelles. 528 p.

Osmaston, H. (1959). Working plan for Bugoma Forest, 1960-1970. Entebbe: Uganda Forest Department, $77 \mathrm{pp}$.

Peh, K.-H., Sonké, B., Séné, O., Djuikouo, M.-N., \& Nguembou, C. (2014). Mixed-Forest Species Establishment in a Monodominant Forest in Central Africa:Implications for Tropical Forest Invasibility. PLoS ONE, 9(5): e97585.doi:10.1371/journal.pone.0097585.

Pelissier, R. (1995). Relations entre l'hétérogénéité spatiale et la dynamique de renouvellement d'une forêt dense humide sempervirente (forêt d'uppangala - ghâts occidentaux de l'inde). Thèse d'Etat, spécialité écologie forestière: Université Claude Bernard - Lyon 1p. 42-44. 
Pélissier, R., Dray, S., \& Sabatier, D. (2002). Within-plot relationships between tree species occurrences and hydrological soil constraints : an example in French Guiana investigated through canonical correlation analysis. Plant Ecology, , 162 ( 2). 143-156.

Picard, N. (1999). Passage d'un modèle individuel à un modèle de distribution de la dynamique forestière : Application à une forêt dense tropicale humide de Guyane française. Thèse de doctorat en Sciences (Spécialité sciences forestières): Ecole nationale du génie rurale des eaux et des forêts. P. 49-54.

Puig, H., Fabre, A., \& Gauquelin, T. (1998). Distribution spatiale des plantules et des jeunes plants d'iryanthera hostmannii (Benth) Warb en forêt tropicale humide de Guyanefrançaise. Life Sciences, 1998 (321): 429-435.

Rollet, B. (1974). L'architecture des forêts denses humides sempervirentes de plaines. Nogent sur marne, France: Centre Technique Forestier Tropical, 298 p.

Sabatier, D. G., Prévost, M., Guillaume, J., G. M., Dosso, M., \& Curmi, P. (1997). The influence of soil cover organization on the floristic and structural heterogeneity of a Guianan rain forest. Plant Ecology, 131 (1). 81-108.

Sheil, D., \& Salim, A. ( 2004). Forest Tree Persistence, Elephants, and Stem Scars. BIOTROPICA, 36(4): 505-521.

Torti, S., Coley, P., \& Kursar, T. (2001). Causes and consequences of monodominance in tropical lowland forests. The American Naturalist, 157(2): 141-153.

Traissac, S. (2003). Dynamique spatiale de Vouacapoua americana (Aublet), arbre de forêt tropicale humide a répartition agrégée. Thèse d'Etat: Université Claude Bernard Lyon 1. P. 35-36.

Van der Velden, N., Slik, J., Y-H., H., Lan, G., Lin, L., Deng, X.-B., et al. (2014). Monodominance of Parashorea chinensis on fertile soils in a Chinese tropical rain forest. Journal of Tropical Ecology, 30:311-322.doi:10.1017/S0266467414000212.

Whitmore, T. (1978). Gaps in the forest canopy. In Tropical trees asliving systems. Tomlinson, P.B. and Zimmermann, M.H. (eds.),675pp. Cambridge University Press, New York, 639-655.

Woolley, L., Henkel, T., \& Sillett, S. (2008). Reiteration in the Monodominant Tropical Tree Dicymbe corymbosa (Caesalpiniaceae) and its Potential Adaptive Significance. BIOTROPICA , 40(1): 32-43.

Zziwa, A., Ziraba, Y., \& Mwakali, J. (2009). Timber use practices in Uganda's building construction industry: current situation and future prospects. The Journal of the Institute of Wood Science , Vol 19, 48-53. DOI: 10.1179/002032009X12536100262475. 